\title{
Indigenous Knowledge and Engaging Marine Fishing Practices in North Coastal Andhra Pradesh
}

\author{
Srinivasu Kodi \\ Department of Anthropology, Andhra University, Visakhapatnam, Andhra Pradesh, India \\ E-mail: anthrosrinu.kodi@gmail.com
}

KEYWORDS Development. Boats. Indigenous Knowledge. Marine Fishing. Nets. Resources

ABSTRACT Fishing is one of the fulltime activity for fishermen which provides livelihood for them. The occupation of fishing has been executed by the engaging indigenous knowledge to thrive their livelihoods. In fact, the earlier studies related to fishing communities are explored through the ethnographic accounts to understand society and culture. The fishing communities are having lower literacy and economic development in the studied area but having wider range of knowledge over the marine ecology and fishing methods is an interesting aspect. This paper provided how the fishing communities are employed different type of fishing nets, boats for fishing expeditions by engaging indigenous knowledge. And, it is also provided the engagement of fishermen's knowledge to identify different school fish by locating fishing grounds in the sea.

\section{INTRODUCTION}

The majority of the earth has been occupied by both salt and fresh water bodies such as sea, rivers, lakes and ponds. The varieties of the living organisms are depending on these water bodies to survive and produce the next generation by utilizing a mixture of existing resources. However, these water bodies act as not only as mineral source and also livelihood particularly to human beings and other living organisms. Hence, fishing is one of traditional activity that promotes livelihood empowerment to the humankind. While practicing fishing as traditional activity, they developed variety of techniques to understand water bodies and in its living organism's behaviours over the time. During developing fishing techniques, they also simultaneously made variety of knowledge systems to acquire grip over living organisms which habituated in the water bodies including both fresh and marine ecosystems. Hence, the knowledge systems played a major role in understanding the locality of both fresh and marine ecosystems through experience of the human beings who depended on it. There are several ethnographic contributions made to understand the dynamic social and cultural aspects of fishing communities over the decades (Firth 1946; Barth 1966; Acheson 1981, 1988, 2003). The anthropologists like Malinowski (1931), Hocart (1937) and others have studied fishing in islands like Trobriand, Eddystone and Andaman respectively.
However, these studies are not focused on fishing and also technology of fishing too. Further, Firth (1946) in his study of Malay fishermen opened a new field in studying the sociology of fishing and of the fisher form as a component of the peasant economy. Further, it is also provided the role of crewmembers in fishing activity and their recruitment process and payments systems which a significant aspect in fishing harvest (Acheson 1981); the depletion of fish stocks and impact of modernization process (Johannes 1978). All of these studies are made significant contributions to studying fishing communities' social/ cultural change by bringing the role of technology and its impact on the fisheries.

The fishing communities are knowledgeable with keen understanding about the marine ecosystems and behaviour of the fish varieties in the sea. The knowledge has been developed over the years by methods of experiment through various forms of fishing methods. The dynamics of knowledge systems are given as an asset by forefathers to next generations to accelerate fishing livelihoods (Dalibandhu and Rama Mohan 2021; Dalibandhu and Sharma 2020b). The marine conservation has given habitation to the marine living organisms and depended on the population. Based on the locality of ecosystems, the human beings adopted different types of sustenance mechanisms for existence of life forms. During this progression, the communities particularly depended on marine fishing which they developed a variety of adaptive survival 
mechanisms by producing knowledge. However, fishermen could able to understand the local marine ecosystems through their knowledge innovations according to marine ecology and its adaptation (Dalibandhu and Rama Mohan 2021). In fact, marine environment habituated to various kinds of species with distinct behaviours. The behaviour of the marine living organisms particularly fish varieties, there is need of techniques, strategies and methods for catching. While searching the school of fish, fishermen take on variety of nets, hooks and lines, gears to catch fish by travelling to longer distances in the sea. There are other few studies which have studied on the fishing communities and their indigenous knowledge dimensions while focusing on fishing practices (Raychaudhuri 1980; Mathur 2008; Chandana 2018; Madhanagopal and Pattanaik 2020).

\section{Objectives}

- To understand how indigenous knowledge engaged for the promoting livelihoods of the fishing communities

- To provide the various nets, boats and cost analysis of the fishing related items

- To know the distribution of fishing grounds, type of fish and seasons/months for the success of fishing expeditions

\section{METHODOLOGY}

The study has been conducted among the Vadabalija and Jalari marine fishing communities regarding the engagement of indigenous knowledge systems. The substantial works have been covered among both the Vadabalija and Jalari communities by covering various social and cultural dimensions such as ritual healing dynamics (Nuckolls 1996; Dalibandhu and Sharma 2020a); the cultural dimension of kinship patterns, gender and economy, mechanization of fishing and its impact on livelihoods (Kodanda Rao 1975; Suryanarayana 1977; Prasada Rao and Yatiraj Kumar 1984; Subba Rao 1986; Sridevi 1989; Dalibandhu and Sharma 2018). For this study, it has been carried out among the rural Jalari (Pudimadaka) and rural Vadabalija (Mukkam); urban Jalari (Peda Jalaripeta) and urban Vadabalija (Vasivanipalem). The data has been collected through the schedules, interviews and group discussions among the fishing communities.
The field insights have been presented in this paper to understand how indigenous knowledge contributed to apprehend the livelihoods and its promotion among the fishing communities.

\section{RESULTS AND DISCUSSION}

The knowledge of the fishing communities has unique for the catching fish and fishing expeditions. They have constructed their knowledge from several generations to catch fish and execute their fishing activities. The knowledge is acquired to utilize appropriately to perform fishing activities for success and avoid occupational hazards. Recent global climatic variations and anthropogenic actions impact the ecological conditions that cause an imbalance in marine biological conservation systems. These systems affect the marine species that are directly impacting the fisher livelihoods. This paper explores the dynamics of the indigenous knowledge systems and their engagement in fishing, which empowers the fisher livelihoods. The fishermen engaged different kinds of nets, boats to catch fish. While fishing expeditions, the fishermen always perceive the local ecological conditions, weather conditions, fish behavior, etc., for the success of fishing; otherwise, there will be a loss during the fishing expeditions. The earlier studies are also similarly focused on the various knowledge practices of the fishing communities. These studies majorly emphasised on the interrogate connection between knowledge and livelihoods that promote fishing economy (Raychaudhuri 1980; Mathur 2008; Chandana Sarma 2018; Dalibandhu and Sharma 2020b; Dalibandhu and Rama Mohan 2021).

\section{Measurement of Nets and Availability of Fish}

The fishermen employed different kinds of nets for fishing based on the month or season. The fishermen are particularly cognisant about the availability of fish varieties by understanding marine ecological conditions. They carry the fishing nets on boat based on the availability of fish in particular season to avoid redundant weight on the boat.

\section{Fishing Grounds and Availability of Fish Varieties}

The fishermen reported that various kinds of fishing grounds and availability of fish varieties. 
Table 1: Distribution of mesh size, length/width of net and type of fish

\begin{tabular}{|c|c|c|c|c|}
\hline S. No. & Name of the net & Mesh size & Length/Width & Type of fish (local names) \\
\hline 1 & Big Net & 4-6 fingers & $1500 \mathrm{mts}$ & Sorra, Konam, Vanjaram, Porava, Tivva, etc. \\
\hline 2 & Iraga Vala & $2-3$ fingers & $\begin{array}{l}01-10-2015 \\
\text { W: } 15-25 \mathrm{mts}\end{array}$ & Savada, Kavvallu, Nettallu,Chinna Royya, etc. \\
\hline 3 & Visuru Vala & $1-1 \frac{1}{2}$ fingers & $\begin{array}{l}6 \mathrm{mts} \\
\mathrm{W}: 2 \mathrm{mts}\end{array}$ & $\begin{array}{l}\text { Bontha, Chinna Kavallu, Tella Goparingalu, } \\
\text { Kanagartalu, etc. }\end{array}$ \\
\hline 4 & Chinna Vaddi Vala & 1 No. & $\begin{array}{l}\mathrm{L}-300 \mathrm{mts} \\
\mathrm{W}-12 \mathrm{mts}\end{array}$ & $\begin{array}{l}\text { Pedda Kavallu, Morava, Chinna Porava, Pedda } \\
\text { Porava, etc. }\end{array}$ \\
\hline 5 & Chinna Kavallu Vala & 1 No & $\begin{array}{l}\mathrm{L}-300 \mathrm{mts} \\
\mathrm{W}-15 \mathrm{mts}\end{array}$ & Chinna Nettallu, Chinna Kavallu, etc. \\
\hline 6 & Nadipi Kavallu Vala & Thumb size & $\begin{array}{l}\mathrm{L}-300 \mathrm{mts} \\
\mathrm{W}-20 \mathrm{mts}\end{array}$ & Pedda Kavallu, Morava, Porava etc \\
\hline 7 & $\begin{array}{l}\text { Pedda KavalluVala/ } \\
\text { PeddaVadi vala }\end{array}$ & $1 \frac{1}{2}-2$ fingers & $\begin{array}{l}\mathrm{L}: 40 \mathrm{mts} \\
\mathrm{W}: 15 \mathrm{mts}\end{array}$ & Pedda Kavallu, Morava, Porava, etc. \\
\hline 8 & Nara Naram Vala & $2 \frac{1}{2}-3$ fingers & $\begin{array}{l}\text { L: } 1050 \mathrm{mts} \\
\mathrm{W}: 15 \mathrm{mts}\end{array}$ & Kanagartalu, Vanjaram, Chinna Sorra etc. \\
\hline 9 & Kadurla Vala & 0 size (3 MS) & $\begin{array}{l}\text { L: } 50 \mathrm{mts} \\
\mathrm{W}: 4 \mathrm{mts}\end{array}$ & Chinna Kavallu, Kadurlu Nettallu, Karalu, etc. \\
\hline 10 & Katta Vala & $1 \frac{1}{2}-2$ fingers & $\begin{array}{l}\text { L: } 1000 \mathrm{mts} \\
\mathrm{W}: 15 \mathrm{mts}\end{array}$ & Kavvallu, Morava, Porava etc. \\
\hline 11 & Alivi Vala & $0 \operatorname{size}(3 \mathrm{MS})$ & $\begin{array}{l}\text { L: } 500 \mathrm{mts} \\
\mathrm{W}: 100 \mathrm{mts}\end{array}$ & $\begin{array}{l}\text { Nettallu, Kavallu, Porava, Konam \& other fish } \\
\text { varieties. }\end{array}$ \\
\hline 12 & Pedda Ringu Vala & $\begin{array}{l}0 \text { No to } 6 \\
\text { finger }\end{array}$ & $\begin{array}{l}\mathrm{L}: 360 \mathrm{mts} \\
\mathrm{W}: 60 \mathrm{mts}\end{array}$ & $\begin{array}{l}\text { Kavvallu, Porava, Chanduva, Royya \& other fish } \\
\text { varieties }\end{array}$ \\
\hline 13 & Pandu Vala / Erra Vala & $5-6$ fingers & $\begin{array}{l}\text { L: } 1500 \mathrm{mts} \\
\mathrm{W}: 12 \mathrm{mts}\end{array}$ & $\begin{array}{l}\text { Konam, Jella, Porava, Royya, Kachchili, Sora \& } \\
\text { other fish varieties }\end{array}$ \\
\hline 14 & Mettani Vala & 0size 2 (MS) & $\begin{array}{l}\text { L: } 50 \mathrm{mts} \\
\mathrm{W}: 4 \mathrm{mts}\end{array}$ & Chinna Kavvallu, Nettallu, etc. \\
\hline 15 & Disco Vala & $2 \frac{1}{2}-4$ fingers & $\begin{array}{l}\text { L: } 50 \mathrm{mts} \\
\mathrm{W}: 4 \mathrm{mts}\end{array}$ & Royya, Peti Royya, Nalla Royya etc \\
\hline 16 & Chandu Vala & 6-7 fingers & $\begin{array}{l}\text { L: } 1000 \mathrm{mts} \\
\mathrm{W}: 15 \mathrm{mts}\end{array}$ & Nalla Chanduva, Sorra, etc. \\
\hline 17 & Nadipi Vaddi Vala & 1 finger & $\begin{array}{l}\text { L: } 140 \mathrm{mts} \\
\mathrm{W}: 10 \mathrm{mts}\end{array}$ & Kavvallu, Nettallu, etc. \\
\hline 18 & Pedda Vaddi Vala & 1-2 fingers & $\begin{array}{l}\mathrm{L}: 270 \mathrm{mts} \\
\mathrm{W}: 24 \mathrm{mts}\end{array}$ & Kanagartalu, Morava, Kavallu, Pedd Parava, etc. \\
\hline 19 & Jogu Vala & 3 fingers & $\begin{array}{l}\mathrm{L}: 500 \mathrm{mts} \\
\mathrm{W}: 11 \mathrm{mts}\end{array}$ & Kanagartalu, Vanjaram,Kalladugu, Sorra, etc. \\
\hline 20 & Chiravaddi Vala & 0 size & $\begin{array}{l}\text { L: } 50 \\
\text { W: } 20\end{array}$ & Chinna Kavallu, Nethallu \\
\hline 21 & Chintha aaku Vala & $\begin{array}{l}1 \text { finger } \\
\text { (Thumb) }\end{array}$ & $\begin{array}{l}\text { L: } 60 \\
\text { W: } 20\end{array}$ & Pedda Kavallu Morava \\
\hline 22 & Iraga vala (Big) & $0-4$ Finger & $\begin{array}{l}\mathrm{L}: 24 \\
\mathrm{~W}: 12\end{array}$ & Savada, Kavallu, Chaduva, Jella, Royya, Peti Royya \\
\hline 23 & Chinna Ringala vala & Size of MS & $\begin{array}{l}\text { L: } 300 \\
\text { W: } 12\end{array}$ & $\begin{array}{l}\text { Kavallu, Nettallu, Kanagarthulu, Poravulu, } \\
\text { Jallalu,Kaliminda, Bonthalu }\end{array}$ \\
\hline 24 & $\begin{array}{l}\text { Middle Size ringu- } \\
\text { lavala }\end{array}$ & 2 finger & $\begin{array}{l}\text { L: } 400 \\
\text { W: } 60\end{array}$ & $\begin{array}{l}\text { Vanjiram, Kanagartalu, Suralu,Chandavalu, Po- } \\
\text { ravalu, Jella }\end{array}$ \\
\hline 25 & Kadurivala & 0/1 Size & $\begin{array}{l}\text { L: } 100 \\
\text { W: } 30\end{array}$ & Kavallu, Kadurulu \\
\hline 26 & Silk vala & 4 finger & $\begin{array}{l}\text { L: } 300 \\
\text { W: } 15\end{array}$ & Porlu, Rai Chepa, Bonthalu, Komalu \\
\hline 27 & Chengula vala & 0 size & $\begin{array}{l}\text { L: } 40 \\
\text { W: } 15\end{array}$ & $\begin{array}{l}\text { Kavallu, Medhadlu, Karalu, Kommu Kara, Palli } \\
\text { Kara, Dasam Kara, Zeerukara }\end{array}$ \\
\hline
\end{tabular}

Anthropologist, 44(1-3): 1-9 (2021) 
Table 1: Cont.....

\begin{tabular}{|c|c|c|c|c|}
\hline S.No. & Name of the net & Mesh size & Length/Width & Type of fish (local names) \\
\hline 28 & Katla vala & 2 finger & $\begin{array}{l}\mathrm{L}: 300 \\
\mathrm{~W}: 200\end{array}$ & $\begin{array}{l}\text { Kavallu, Badhakavallu, Moravalu, Korava, Bur- } \\
\text { rakavallu, Pilladugu, Chinnasora, Chinku, Royya, } \\
\text { Tiger Royya, Peti Royya }\end{array}$ \\
\hline 29 & Pedda Nabu vala & 10 finger & $\begin{array}{l}\text { L: } 1000 \\
W: 20\end{array}$ & Pandu Kappa, Goraka, Suralu \\
\hline 30 & Maaga vala & 5 fingers & $\begin{array}{l}\mathrm{L}: 1500 \\
\mathrm{~W}: 12\end{array}$ & Maaga, Porava, Konam, Suralu \\
\hline
\end{tabular}

Source: Fieldwork

Table 2: Measurement of fishing boats and its approximate cost

\begin{tabular}{|c|c|c|c|c|c|c|c|c|c|c|c|}
\hline \multirow{2}{*}{$\begin{array}{l}S . \\
\text { No. }\end{array}$} & \multirow{2}{*}{ Type of boat } & \multirow{2}{*}{$\begin{array}{l}\text { Boat cost } \\
\text { (Approxi- } \\
\text { mate) }\end{array}$} & \multirow{2}{*}{$\begin{array}{c}\text { Engine } \\
\text { cost } \\
\text { (Approxi- } \\
\text { mate) }\end{array}$} & \multirow{2}{*}{$\begin{array}{c}\text { Front end to } \\
\text { backend }\end{array}$} & \multicolumn{4}{|c|}{ Length } & \multicolumn{3}{|c|}{ Width } \\
\hline & & & & & $\begin{array}{l}\text { At the } \\
\text { centre of } \\
\text { the boat }\end{array}$ & $\begin{array}{c}\text { At } \\
1 \mathrm{~m} \text { from } \\
\text { front end }\end{array}$ & $\begin{array}{c}\text { At } \\
\text { I m from } \\
\text { back end }\end{array}$ & $\begin{array}{c}\text { At back } \\
\text { end fibre } \\
\text { boat }\end{array}$ & $\begin{array}{l}\text { At front } \\
\text { end }\end{array}$ & $\begin{array}{l}\text { At the } \\
\text { centre }\end{array}$ & $\begin{array}{c}\text { At the } \\
\text { back } \\
\text { end }\end{array}$ \\
\hline \multirow[t]{2}{*}{1} & $\begin{array}{l}\text { Country boat } \\
\text { (S) }\end{array}$ & 12000 & - & 12.08 & 3.06 & 2.06 & 3.01 & 2.03 & 0.02 & 0.07 & 0.06 \\
\hline & & & & 3.86 & 1.06 & 0.76 & 0.94 & 0.7 & 0.06 & 0.18 & 0.16 \\
\hline \multirow[t]{2}{*}{2} & $\begin{array}{l}\text { Country boat } \\
\text { (M) }\end{array}$ & 24000 & - & 16 & 4.01 & 2.7 & 3.06 & 2.06 & 0.4 & 1.08 & 1.2 \\
\hline & & & & 4.87 & 1.25 & 0.8 & 1.07 & 0.75 & 0.11 & 0.5 & 0.37 \\
\hline \multirow[t]{2}{*}{3} & $\begin{array}{l}\text { Country boat } \\
\text { (M) }\end{array}$ & 24000 & - & 16 & 3.03 & 2 & 3.01 & 2.06 & 0.6 & 1.04 & 1.1 \\
\hline & & & & 4.87 & 0.98 & 0.6 & 0.95 & 0.77 & 0.16 & 0.4 & 0.34 \\
\hline \multirow[t]{2}{*}{4} & $\begin{array}{l}\text { Country boat } \\
\text { (M) }\end{array}$ & 24000 & - & 16.03 & 3.3 & 2.1 & 3.1 & 2.06 & 0.06 & 1.04 & 1.02 \\
\hline & & & & 4.95 & 1 & 0.65 & 0.95 & 0.76 & 0.15 & 0.4 & 0.36 \\
\hline \multirow[t]{2}{*}{5} & $\begin{array}{l}\text { Country boat } \\
\text { (M) }\end{array}$ & 26000 & - & 17.5 & 4.1 & 2.02 & 3.01 & 1.12 & 0.7 & 0.08 & 0.1 \\
\hline & & & & 5.31 & 1.5 & 0.68 & 1 & 0.62 & 0.16 & 0.2 & 0.25 \\
\hline \multirow[t]{2}{*}{6} & $\begin{array}{l}\text { Country boat } \\
\text { (S) FI }\end{array}$ & 45000 & 30000 & 14 & 3.9 & 2.04 & 0.32 & 1.08 & 0.8 & 1.4 & 1.2 \\
\hline & & & & 5.5 & 1.15 & 0.72 & 0.96 & 0.6 & 0.22 & 0.4 & 0.3 \\
\hline \multirow[t]{2}{*}{7} & $\begin{array}{l}\text { Country boat } \\
\text { (M) FI }\end{array}$ & 45000 & 30000 & 16.08 & 3.05 & 2.2 & 3.2 & 2.08 & 0.06 & 1.06 & 1.04 \\
\hline & & & & 5.39 & 1.02 & 0.67 & 0.98 & 0.78 & 0.15 & 0.42 & 0.38 \\
\hline \multirow[t]{2}{*}{8} & $\begin{array}{l}\text { Country boat } \\
\text { (M) FI }\end{array}$ & 42000 & 30000 & 17.9 & 3.2 & 2.1 & 3.01 & 2.01 & 1.18 & 0.12 & 1.05 \\
\hline & & & & 5.41 & 1.02 & 0.52 & 0.91 & 0.64 & 0.67 & 0.35 & 0.44 \\
\hline \multirow[t]{2}{*}{9} & $\begin{array}{l}\text { Country boat } \\
\text { (M) FI }\end{array}$ & 670000 & 30000 & 18.06 & 3.08 & 2.1 & 2.1 & 1.12 & 0.6 & 0.8 & 0.9 \\
\hline & & & & 5.64 & 1.12 & 0.65 & 0.87 & 0.62 & 0.15 & 0.2 & 0.22 \\
\hline \multirow[t]{2}{*}{10} & $\begin{array}{l}\text { Country boat } \\
\text { (M) FI }\end{array}$ & 670000 & 45000 & 18.8 & 3.5 & 2.1 & 3 & 2.01 & 0.3 & 2.01 & 1.06 \\
\hline & & & & 5.7 & 1.9 & 0.62 & 0.9 & 0.64 & 0.7 & 0.7 & 0.48 \\
\hline \multirow[t]{2}{*}{11} & $\begin{array}{l}\text { Country boat } \\
\text { (M) FI }\end{array}$ & 670000 & 45000 & 18.1 & 4.13 & 2.3 & 3.06 & 2.04 & 0.05 & 0.08 & 0.1 \\
\hline & & & & 5.74 & 1.32 & 0.68 & 1.02 & 0.7 & 0.1 & 0.1 & 0.25 \\
\hline \multirow[t]{2}{*}{12} & $\begin{array}{l}\text { Country boat } \\
\text { (M) FI }\end{array}$ & 670000 & 55000 & 18.02 & 3.8 & 2.04 & 3.1 & 2.02 & 0.2 & 2.05 & 1.08 \\
\hline & & & & 5.54 & 1.13 & 0.71 & 0.95 & 0.66 & 0.6 & 0.73 & 0.51 \\
\hline 13 & $\begin{array}{l}\text { Country boat } \\
\text { (M) FI }\end{array}$ & 90000 & 60000 & 18.11 & 4.32 & 2.3 & 3.05 & 2.04 & 0.05 & 0.1 & 0.1 \\
\hline
\end{tabular}

Anthropologist, 44(1-3): 1-9 (2021) 
Table 2: Cont .....

\begin{tabular}{|c|c|c|c|c|c|c|c|c|c|c|c|}
\hline \multirow{2}{*}{$\begin{array}{l}S . \\
\text { No. }\end{array}$} & \multirow{2}{*}{ Type of boat } & \multirow{2}{*}{$\begin{array}{l}\text { Boat cost } \\
\text { (Approxi- } \\
\text { mate) }\end{array}$} & \multirow{2}{*}{$\begin{array}{c}\text { Engine } \\
\text { cost } \\
\text { (Approxi- } \\
\text { mate) }\end{array}$} & \multirow{2}{*}{$\begin{array}{l}\text { Front end to } \\
\text { backend }\end{array}$} & \multicolumn{4}{|c|}{ Length } & \multicolumn{3}{|c|}{ Width } \\
\hline & & & & & $\begin{array}{l}\text { At the } \\
\text { centre of } \\
\text { the boat }\end{array}$ & $\begin{array}{c}A t \\
1 \mathrm{~m} \text { from } \\
\text { front end }\end{array}$ & $\begin{array}{c}\text { At } \\
\text { I m from } \\
\text { back end }\end{array}$ & $\begin{array}{c}\text { At back } \\
\text { end fibre } \\
\text { boat }\end{array}$ & $\begin{array}{l}\text { At front } \\
\text { end }\end{array}$ & $\begin{array}{l}\text { At the } \\
\text { centre }\end{array}$ & $\begin{array}{c}\text { At the } \\
\text { back } \\
\text { end }\end{array}$ \\
\hline \multirow[t]{2}{*}{14} & $\begin{array}{l}\text { Country boat } \\
\text { (M) FI }\end{array}$ & 100000 & 60000 & 19.07 & 3.1 & 2.03 & 3.04 & 2.5 & 0.05 & 0.9 & 0.1 \\
\hline & & & & 5.97 & 1.16 & 0.68 & 1 & 0.75 & 0.1 & 0.24 & 0.25 \\
\hline \multirow[t]{2}{*}{15} & $\begin{array}{l}\text { Country boat } \\
\text { (M) FI }\end{array}$ & 100000 & 60000 & 19.1 & 4.01 & 2.05 & 3.05 & 2.7 & 0.07 & 0.1 & 0.1 \\
\hline & & & & 6 & 1.25 & 0.74 & 1 & 0.8 & 0.18 & 0.25 & 0.25 \\
\hline \multirow[t]{2}{*}{16} & $\begin{array}{l}\text { Country boat } \\
\text { (L) FI }\end{array}$ & 110000 & 60000 & 20.2 & 3.8 & 2 & 3.02 & 2.09 & 0.7 & 1.1 & 1 \\
\hline & & & & 6.15 & 1.12 & 0.61 & 0.97 & 0.84 & 0.18 & 0.34 & 0.32 \\
\hline \multirow[t]{2}{*}{17} & $\begin{array}{l}\text { Country boat } \\
\text { (L) FI }\end{array}$ & 110000 & 60000 & 20.2 & 3.8 & 2.03 & 2.08 & 2.8 & 0.6 & 1.1 & 1 \\
\hline & & & 60000 & 6.15 & 1.1 & 0.69 & 0.81 & 0.82 & 0.16 & 0.32 & 0.3 \\
\hline \multirow[t]{2}{*}{18} & $\begin{array}{l}\text { Country boat } \\
\text { (L) Eng }\end{array}$ & 130000 & 60000 & 21.06 & 3.1 & 2 & 3 & 2.09 & 0.7 & 1.02 & 1.01 \\
\hline & & & & 6.55 & 1.17 & 0.61 & 0.92 & 0.84 & 0.18 & 0.36 & 0.26 \\
\hline \multirow[t]{2}{*}{19} & $\begin{array}{l}\text { Country boat } \\
\text { (L) Eng }\end{array}$ & 140000 & 60000 & 23.1 & 4.25 & 2.03 & 2.08 & 2.02 & 0.7 & 1.02 & 1 \\
\hline & & & & 7.25 & 4.1 & 0.67 & 0.82 & 0.66 & 0.19 & 0.36 & 0.3 \\
\hline \multirow[t]{2}{*}{20} & $\begin{array}{l}\text { Country } \\
\text { boat(L)Eng }\end{array}$ & 150000 & 60000 & 25.11 & 4.04 & 2.01 & 3.4 & 2.5 & 0.08 & 1.04 & 1 \\
\hline & & & & 7.9 & 1.31 & 0.65 & 1.03 & 0.76 & 0.2 & 1.41 & 0.34 \\
\hline \multirow[t]{2}{*}{21} & $\begin{array}{l}\text { Kuttupadava } \\
\text { (M) Eng }\end{array}$ & 25000 & 45000 & 16 & 4.01 & 2.7 & 3.06 & 2.06 & 0.4 & 1.08 & 1.2 \\
\hline & & & & 4.87 & 1.25 & 0.8 & 1.07 & 0.75 & 0.11 & 0.5 & 0.37 \\
\hline \multirow[t]{2}{*}{22} & $\begin{array}{l}\text { Kuttupadava } \\
\text { (M) Eng }\end{array}$ & 27000 & 45000 & 16.08 & 3.6 & 2.1 & 3.4 & 2.11 & 0.4 & 1.03 & 1.2 \\
\hline & & & & 5.08 & 1.07 & 0.65 & 1.03 & 0.89 & 0.11 & 0.37 & 0.37 \\
\hline \multirow[t]{2}{*}{23} & $\begin{array}{l}\text { Kuttupadava } \\
\text { (M) Eng }\end{array}$ & 30000 & 60000 & 18 & 3.4 & 2 & 2.11 & 2.04 & 0.2 & 0.7 & 1 \\
\hline & & & & 5.49 & 1.02 & 0.6 & 0.9 & 0.71 & 0.01 & 0.18 & 0.3 \\
\hline \multirow[t]{2}{*}{24} & $\begin{array}{l}\text { Kuttupadava } \\
\text { (M) Eng }\end{array}$ & 30000 & 60000 & 18.1 & 3.07 & 1.1 & 2.11 & 2.02 & 0.02 & 0.07 & 0.8 \\
\hline & & & & 5.55 & 1.1 & 0.57 & 0.87 & 0.6 & 0 & 0.17 & 0.21 \\
\hline \multirow[t]{2}{*}{25} & $\begin{array}{l}\text { Kuttupadava } \\
\text { (L) Eng }\end{array}$ & 35000 & 60000 & 21.2 & 3.8 & 2.09 & 2.07 & 2.04 & 0.6 & 1.02 & 2.04 \\
\hline & & & & 6.46 & 1.14 & 0.84 & 0.79 & 0.81 & 0.18 & 0.35 & 0.7 \\
\hline \multirow[t]{2}{*}{26} & $\begin{array}{l}\text { Kuttupadava } \\
\text { (L) Eng }\end{array}$ & 42000 & 60000 & 24.02 & 3.1 & 2 & 3.04 & 2.09 & 0.8 & 1.04 & 1.03 \\
\hline & & & & 7.37 & 1.2 & 0.61 & 1 & 0.85 & 0.22 & 0.42 & 0.38 \\
\hline \multirow[t]{2}{*}{27} & $\begin{array}{l}\text { Kuttupadava } \\
\text { (L) Eng }\end{array}$ & 25000 & 30000 & 16.07 & 3.3 & 3 & 3.5 & 2.05 & 0.6 & 1.04 & 1.02 \\
\hline & & & & 4.92 & 1 & 3.64 & 0.68 & 0.75 & 0.15 & 0.4 & 0.06 \\
\hline \multirow[t]{2}{*}{28} & $\begin{array}{l}\text { Kuttupadava } \\
\text { (L) Eng }\end{array}$ & 45000 & 55000 & 26.07 & 5.8 & 2.05 & 3 & 0.1 & 2 & 3.2 & 1.1 \\
\hline & & & & 8.1 & 1.74 & 0.74 & 0.92 & 0.25 & 0.61 & 0.98 & 0.56 \\
\hline \multirow[t]{2}{*}{29} & $\begin{array}{l}\text { Kuttupadava } \\
\text { (L)Eng }\end{array}$ & 48000 & 60000 & 29.5 & 4.03 & 2.03 & 2.06 & 0.1 & 0.7 & 1.04 & 1.2 \\
\hline & & & & 8.95 & 1.3 & 0.95 & 0.758 & 0.26 & 0.18 & 0.4 & 0.35 \\
\hline \multirow[t]{2}{*}{30} & $\begin{array}{l}\text { Kuttupadava } \\
\text { (L)Eng }\end{array}$ & 48000 & 60000 & 28.05 & 6.04 & 3.02 & 5.07 & 3.09 & 3.06 & 1.1 & 2 \\
\hline & & & & 8.62 & 1.94 & 0.95 & 1.7 & 1.14 & 1.06 & 0.56 & 0.61 \\
\hline
\end{tabular}

Anthropologist, 44(1-3): 1-9 (2021) 
Table 2: Cont.....

\begin{tabular}{|c|c|c|c|c|c|c|c|c|c|c|c|}
\hline \multirow{2}{*}{$\begin{array}{l}S . \\
\text { No. }\end{array}$} & \multirow{2}{*}{ Type of boat } & \multirow{2}{*}{$\begin{array}{l}\text { Boat cost } \\
\text { (Approxi- } \\
\text { mate) }\end{array}$} & \multirow{2}{*}{$\begin{array}{c}\text { Engine } \\
\text { cost } \\
\text { (Approxi- } \\
\text { mate) }\end{array}$} & \multirow{2}{*}{$\begin{array}{c}\text { Front end to } \\
\text { backend }\end{array}$} & \multicolumn{4}{|c|}{ Length } & \multicolumn{3}{|c|}{ Width } \\
\hline & & & & & $\begin{array}{l}\text { At the } \\
\text { centre of } \\
\text { the boat }\end{array}$ & $\begin{array}{c}\text { At } \\
1 \mathrm{~m} \text { from } \\
\text { front end }\end{array}$ & $\begin{array}{c}\text { At } \\
\text { I m from } \\
\text { back end }\end{array}$ & $\begin{array}{c}\text { At back } \\
\text { end fibre } \\
\text { boat }\end{array}$ & $\begin{array}{l}\text { At front } \\
\text { end }\end{array}$ & $\begin{array}{l}\text { At the } \\
\text { centre }\end{array}$ & $\begin{array}{c}\text { At the } \\
\text { back } \\
\text { end }\end{array}$ \\
\hline \multirow[t]{2}{*}{31} & $\begin{array}{l}\text { Kuttupadava } \\
\text { (L) Eng }\end{array}$ & 49000 & 60000 & 29.6 & 6.06 & 1.9 & 2.11 & 0.08 & 2.04 & 3.07 & 1.03 \\
\hline & & & & 9 & 1.98 & 0.55 & 0.9 & 0.2 & 2.71 & 1.1 & 0.39 \\
\hline \multirow[t]{2}{*}{32} & $\begin{array}{l}\text { Kuttu boat(L) } \\
\text { FI (Eng) }\end{array}$ & 50000 & 60000 & 29.1 & 4.4 & 3.3 & 2.1 & 3.02 & 0.2 & 1.05 & 1.02 \\
\hline & & & & 9.1 & 1.32 & 1.5 & 0.85 & 0.96 & 0.8 & 0.44 & 0.35 \\
\hline 33 & $\begin{array}{l}\text { Kuttu boat } \\
\text { (L) FI (Eng) }\end{array}$ & 55000 & 60000 & 34.6 & 4.6 & 0.11 & 3.1 & 2.04 & 0.9 & 1.05 & 1.2 \\
\hline \multirow[t]{2}{*}{34} & $\begin{array}{l}\text { Kuttu boat (L) } \\
\text { Eng }\end{array}$ & 55000 & 60000 & 38.1 & 4.06 & 1.7 & 2.11 & 2.04 & 0.07 & 1.06 & 1.03 \\
\hline & & & & 11.83 & 1.37 & 0.49 & 0.9 & 0.71 & 0.18 & 0.86 & 0.38 \\
\hline \multirow[t]{2}{*}{35} & $\begin{array}{l}\text { Kuttu boat (L) } \\
\text { Eng }\end{array}$ & 55000 & 60000 & 38.4 & 4.1 & 2 & 3.06 & 3.02 & 0.09 & 1.9 & 1.5 \\
\hline & & & & 11.7 & 1.49 & 0.62 & 1.07 & 0.87 & 0.22 & 0.54 & 0.44 \\
\hline \multirow[t]{2}{*}{36} & $\begin{array}{l}\text { Kuttu boat (L) } \\
\text { Eng }\end{array}$ & 58000 & 60000 & 39.1 & 4.08 & 2.01 & 3.05 & 2.11 & 0.22 & 0.52 & 1.4 \\
\hline & & & & 12.14 & 1.42 & 0.62 & 1.03 & 0.89 & 0.09 & 1.08 & 0.4 \\
\hline \multirow[t]{2}{*}{37} & $\begin{array}{l}\text { Terachapa(L) } \\
\text { En }\end{array}$ & 160000 & 60000 & 34.4 & 4.9 & 1.3 & 3.6 & 0.09 & 2.06 & 3.09 & 1.06 \\
\hline & & & & 10.5 & 1.45 & 4.3 & 1.7 & 0.22 & 0.72 & 1.12 & 0.42 \\
\hline \multirow[t]{2}{*}{38} & $\begin{array}{l}\text { Terachapa(L) } \\
\text { En }\end{array}$ & 145000 & 60000 & 29.11 & 4.06 & 3.06 & 2.11 & 3.08 & 0.2 & 1.5 & 0.35 \\
\hline & & & & 9.12 & 1.37 & 1.07 & 0.86 & 1.7 & 0.8 & 0.44 & 1.2 \\
\hline \multirow[t]{2}{*}{39} & $\begin{array}{l}\text { Terachapa(L) } \\
\text { En }\end{array}$ & 55000 & 45000 & 16.03 & 3.3 & 2.01 & 3.2 & 2.08 & 0.06 & 1.06 & 1.04 \\
\hline & & & & 4.95 & 1.3 & 0.66 & 0.98 & 0.77 & 0.15 & 0.42 & 0.38 \\
\hline \multirow[t]{2}{*}{40} & $\begin{array}{l}\text { Terachapa(L) } \\
\text { En }\end{array}$ & 180000 & 60000 & 44.3 & 4.27 & 1.82 & 3.1 & 0.8 & 0.1 & 1.6 & 1.45 \\
\hline & & & & 13.42 & 1.4 & 0.54 & 0.94 & 2.04 & 0.18 & 0.42 & 0.42 \\
\hline \multirow[t]{2}{*}{41} & $\begin{array}{l}\text { Fiber boat }(\mathrm{L}) \\
\text { Eng }\end{array}$ & 160000 & 60000 & 29.04 & 7.1 & 3.02 & 5.07 & 3.09 & 3.06 & 1.1 & 2 \\
\hline & & & & 8.94 & 2.4 & 0.95 & 1.7 & 1.14 & 1.06 & 0.56 & 0.61 \\
\hline \multirow[t]{2}{*}{42} & $\begin{array}{l}\text { Fiber boat(L) } \\
\text { Eng }\end{array}$ & 160000 & 60000 & 29.4 & 7.1 & 3.1 & 5.6 & 3.09 & 3.03 & 1.1 & 2 \\
\hline & & & & 8.95 & 2.39 & 1.17 & 1.68 & 1.14 & 1 & 0.5 & 0.61 \\
\hline \multirow[t]{2}{*}{43} & $\begin{array}{l}\text { Fiber boat(L) } \\
\text { FI(Eng) }\end{array}$ & 160000 & 60000 & 29.4 & 8 & 3.1 & 5.6 & 3.09 & 3.3 & 1.08 & 1.1 \\
\hline & & & & 8.95 & 2.44 & 1.17 & 1.68 & 1.14 & 1 & 0.5 & 0.6 \\
\hline \multirow[t]{2}{*}{44} & $\begin{array}{l}\text { Fiber boat(L) } \\
\text { FI(Eng) }\end{array}$ & 158000 & 60000 & 28.1 & 4.05 & 2.01 & 3.04 & 2.11 & 0.99 & 1.05 & 1.04 \\
\hline & & & & 8.82 & 0.35 & 0.63 & 1.02 & 0.9 & 0.23 & 0.43 & 0.4 \\
\hline \multirow[t]{2}{*}{45} & $\begin{array}{l}\text { Fiber boat(L) } \\
\text { Eng }\end{array}$ & 160000 & 60000 & 29.1 & 4.06 & 3.06 & 2.11 & 3.05 & 0.2 & 1.5 & 1.04 \\
\hline & & & & 9.12 & 1.37 & 1.07 & 0.86 & 1.05 & 1.08 & 0.44 & 0.37 \\
\hline \multirow[t]{2}{*}{46} & $\begin{array}{l}\text { Fiber boat(L) } \\
\text { Eng }\end{array}$ & 165000 & 60000 & 33.1 & 4.06 & 2.03 & 2.06 & 2.9 & 0.08 & 1.03 & 1.02 \\
\hline & & & & 10.3 & 1.36 & 0.95 & 0.75 & 0.85 & 0.3 & 0.37 & 0.35 \\
\hline \multirow[t]{2}{*}{47} & $\begin{array}{l}\text { Fiber boat }(\mathrm{L}) \\
\text { Eng }\end{array}$ & 170000 & 60000 & 35 & 4.06 & 1.11 & 2.11 & 2.3 & 0.06 & 1.4 & 1.03 \\
\hline & & & & 10.67 & 1.39 & 0.52 & 0.89 & 0.7 & 0.16 & 0.42 & 0.37 \\
\hline \multirow[t]{2}{*}{48} & $\begin{array}{l}\text { Fiber boat }(\mathrm{L}) \\
\text { Eng }\end{array}$ & 180000 & 60000 & 36.01 & 10.1 & 3.3 & 7.7 & 6 & 1 & 3.11 & 2.08 \\
\hline & & & & 11 & 3.17 & 1 & 3.32 & 1.84 & 0.31 & 1.2 & 0.85 \\
\hline
\end{tabular}

Anthropologist, 44(1-3): 1-9 (2021) 


\begin{tabular}{|c|c|c|c|c|c|c|c|c|c|c|c|}
\hline \multirow{2}{*}{$\begin{array}{l}\text { S. } \\
\text { No. }\end{array}$} & \multirow{2}{*}{ Type of boat } & \multirow{2}{*}{$\begin{array}{l}\text { Boat cost } \\
\text { (Approxi- } \\
\text { mate) }\end{array}$} & \multirow{2}{*}{$\begin{array}{c}\text { Engine } \\
\text { cost } \\
\text { (Approxi- } \\
\text { mate) }\end{array}$} & \multirow{2}{*}{$\begin{array}{c}\text { Front end to } \\
\text { backend }\end{array}$} & \multicolumn{4}{|c|}{ Length } & \multicolumn{3}{|c|}{ Width } \\
\hline & & & & & $\begin{array}{l}\text { At the } \\
\text { centre of } \\
\text { the boat }\end{array}$ & $\begin{array}{c}\text { At } \\
1 \mathrm{~m} \text { from } \\
\text { front end }\end{array}$ & $\begin{array}{c}\text { At } \\
\text { I m from } \\
\text { back end }\end{array}$ & $\begin{array}{c}\text { At back } \\
\text { end fibre } \\
\text { boat }\end{array}$ & $\begin{array}{c}\text { At front } \\
\text { end }\end{array}$ & $\begin{array}{l}\text { At the } \\
\text { centre }\end{array}$ & $\begin{array}{l}\text { At the } \\
\text { back } \\
\text { end }\end{array}$ \\
\hline \multirow[t]{2}{*}{49} & $\begin{array}{l}\text { Fiber boat(L) } \\
\text { Eng }\end{array}$ & 180000 & 60000 & 36.01 & 4.8 & 2.01 & 3.1 & 2.03 & 0.06 & 1.06 & 1.04 \\
\hline & & & & 11 & 1.43 & 0.63 & 0.95 & 0.68 & 0.23 & 0.46 & 0.4 \\
\hline \multirow[t]{2}{*}{50} & $\begin{array}{l}\text { Fiber boat }(\mathrm{L}) \\
\text { Eng }\end{array}$ & 200000 & 60000 & 38.1 & 4.3 & 3.01 & 2.9 & 2.03 & 0.09 & 1.05 & 1.02 \\
\hline & & & & 11.8 & 1.4 & 0.95 & 0.85 & 0.69 & 0.23 & 0.45 & 0.35 \\
\hline \multirow[t]{2}{*}{51} & $\begin{array}{l}\text { Fiber boat }(\mathrm{L}) \\
\text { Eng }\end{array}$ & 210000 & 60000 & 41.5 & 4.5 & 3.6 & 3.01 & 2.08 & 0.11 & 1.07 & 1.06 \\
\hline & & & & 12.5 & 1.5 & 1.06 & 0.92 & 0.82 & 0.28 & 0.49 & 0.42 \\
\hline \multirow[t]{2}{*}{52} & $\begin{array}{l}\text { Fiber boat(L) } \\
\text { Eng }\end{array}$ & 215000 & 60000 & 42.3 & 4.07 & 1.09 & 3.01 & 2.07 & 0.67 & 1.06 & 1.45 \\
\hline & & & & 13.48 & 1.4 & 0.54 & 0.94 & 0.8 & 0.18 & 0.45 & 0.42 \\
\hline \multirow[t]{2}{*}{53} & $\begin{array}{l}\text { Fiber boat( }(\mathrm{L}) \\
\text { Eng }\end{array}$ & 220000 & 60000 & 44.1 & 4.09 & 1.11 & 3.01 & 2.06 & 0.08 & 1.08 & 1.04 \\
\hline & & & & 13.41 & 1.44 & 0.59 & 0.94 & 0.79 & 0.2 & 0.5 & 0.4 \\
\hline \multirow[t]{2}{*}{54} & $\begin{array}{l}\text { Fiber boat }(\mathrm{L}) \\
\text { Eng }\end{array}$ & 223000 & 60000 & 44.1 & 4.07 & 2 & 3.02 & 2.7 & 0.1 & 1.6 & 1.04 \\
\hline & & & & 13.67 & 1.39 & 0.61 & 0.96 & 0.8 & 0.26 & 0.47 & 0.4 \\
\hline \multirow[t]{2}{*}{55} & $\begin{array}{l}\text { Fiber boat(L) } \\
\text { Eng }\end{array}$ & 230000 & 60000 & 46.4 & 6.7 & 2.04 & 9.6 & 3.1 & 0.1 & 2.02 & 1.1 \\
\hline & & & & 14.1 & 2 & 0.7 & 0.46 & 0.9 & 0.26 & 0.66 & 0.57 \\
\hline \multirow[t]{3}{*}{56} & $\begin{array}{l}\text { Fiber boat }(\mathrm{L}) \\
\text { Eng }\end{array}$ & 235000 & 60000 & 48 & 6.07 & 2.04 & 9.6 & 3.1 & 0.1 & 2.02 & 1.1 \\
\hline & & & & 14.63 & 2 & 0.7 & 0.46 & 0.94 & & 0.66 & 0.57 \\
\hline & & & & 15.28 & 2 & 0.7 & 6.9 & 0.94 & 0.26 & 0.66 & 0.57 \\
\hline \multirow[t]{2}{*}{58} & $\begin{array}{l}\text { Fiber boat(L) } \\
\text { Eng }\end{array}$ & 235000 & 60000 & 47.06 & 4.09 & 2 & 3.01 & 2.06 & 0.08 & 1.6 & 1.4 \\
\hline & & & & 14.48 & 1.44 & 0.91 & 0.94 & 0.76 & 0.2 & 0.47 & 0.4 \\
\hline \multirow[t]{2}{*}{59} & $\begin{array}{l}\text { Fiber boat }(\mathrm{L}) \\
\text { Eng }\end{array}$ & 250000 & 60000 & 48.04 & 4.09 & 2.02 & 3.02 & 2.06 & 0.08 & 1.9 & 1.4 \\
\hline & & & & 15.04 & 1.44 & 0.63 & 0.95 & 0.79 & 0.2 & 0.47 & 0.4 \\
\hline \multirow[t]{2}{*}{60} & $\begin{array}{l}\text { Fiber boat(L) } \\
\text { Eng }\end{array}$ & 260000 & 60000 & 52 & 6.07 & 2.04 & 6.9 & 3.1 & 0.1 & 2.02 & 1.1 \\
\hline & & & & 15.85 & 2 & 0.7 & 6.9 & 0.94 & 0.26 & 0.66 & 0.57 \\
\hline
\end{tabular}

Source: Fieldwork

There are several fishing grounds which are present in the sea but only some of them are reported by the fishermen. Based on the season or month, the fishermen go for fishing with their fishing nets, hooks and lines to catch the fish.

\section{Seawater, Tides and Fishing}

The fishermen developed their knowledge over the time by experiencing various kinds of fishing methodologies. The fishermen argued that the knowledge over the sea had an advantage to catch fish an easy way and reduce their physical activity by minimizing manpower. The fishermen identify some kind of light between two tides which they called as manta kavuru. During mantu kavuru, the fishermen will not prefer for fishing with cast net (visuru vala). When there is no manta kavuru in tides, the fishermen prefer to go for cast net because the fish cannot able to see the net. While observing the tides and its interactions, the fishermen can able to understand the fish behaviour and its identification.

The fishermen have knowledge in identifying the fish varieties by observing the marks in the sea which they referred as erra kattindi. Based on these marks, the fishermen cast their nets by

Anthropologist, 44(1-3): 1-9 (2021) 
Table 3: Distribution of fishing grounds, type of fish and seasons/months

\begin{tabular}{|c|c|c|}
\hline Fishing grounds & Type of fish (Local names) & Seasons/Months \\
\hline $\begin{array}{l}\text { Gyla Matta, Budatha Metta, Desam Metta, } \\
\text { Chencheti Konda }\end{array}$ & Kommu Konam, Rekka Sura & June-August \\
\hline Gadil Konda, Pechchidi Konda, Dibba Konda & Kommu Konam, Tuna Fish & $\begin{array}{l}\text { June-July/ November- } \\
\text { December }\end{array}$ \\
\hline Eththu Konda, Pilla Gudi, Sudi Metta & $\begin{array}{l}\text { Kommu Konam, Tuna Fish, Jalagulu, } \\
\text { Avulosulu }\end{array}$ & $\begin{array}{l}\text { June-July/November- } \\
\text { December }\end{array}$ \\
\hline $\begin{array}{l}\text { Oota Gadda, Pandi Dibba, Donkada, Bandanam } \\
\text { Metta, Kota Rayi Konda }\end{array}$ & $\begin{array}{l}\text { Rekka Sura, Kommu Konam, Tuna } \\
\text { Fish }\end{array}$ & $\begin{array}{l}\text { June-July/November- } \\
\text { December }\end{array}$ \\
\hline Pandi Dindi, Etlu Konda, Pitta Konda, Chinna Tella Metta & Kommu Konam, Avulosulu & February-April \\
\hline $\begin{array}{l}\text { Nalla Tedi Konda, Pedda Tella Metta, Nalla Konda, } \\
\text { Dari Metta, Madda Metta }\end{array}$ & $\begin{array}{l}\text { Kommu Konam, Suralu, } \\
\text { Avulosulu }\end{array}$ & November-June \\
\hline Vasanala Paadu, Pedda Naadi Konda, Nalanaadi Konda & Suralu, Avulosulu, Kommi Konam & July-September \\
\hline Pedda Naara, Rendu Nela Konda & Kommu Konam, Tella Sora, Erra Sora & October-April \\
\hline Mudu Naala Konda, Konda Mekalu & Vanjarumu, Avulosulu & February-May \\
\hline Tella Dibbalu & Bukkadugu & October-February \\
\hline Korai Konda,Gedda Konda & Erra Suralu, Bukkadugu & November-April \\
\hline Vankara Dinkara Konda, Chaparaya Metta & Suralu, Vanjaralu & November-June \\
\hline Kapidi Konda & Gopiringalu, Sura & May-August \\
\hline Rushi Konda,Kappera Konda & Sura, Kanartalu & November--June \\
\hline Thamberu Metta, Uppada Konda & Morava, Kavvallu & November-June \\
\hline
\end{tabular}

Source: Fieldwork

covering those marks in the sea. For identifying these marks, the fishermen eyes acts as camera lens to capture the location of school of fish in the sea.

The fishermen reported there are two kinds of layers on the sea tides, that is, outer layer and inner layer. The outer layer of the tides called as katu burada neeru and inner layer of tides named as mucchu neeru. The fishermen believed that the fish are available in the inner layer of the sea tide. The fishermen are expertise in identifying variation between tide's outer layer and inner layer of seawater. Based on these layers of seawater, the fishermen cast their nets to catch the fish. It says that while going to deep sea, if we can find the black colour (nalla neeru) seawater, and then there will be high chance of getting fish varieties such as konalu, kommi konalu, vanjaralu, sorralu etc.

The fishermen referred tides in their local names as teralu, mojulu or keratalu. Based on the height of tides, the fishermen assess the weather conditions and make decisions for fishing expeditions. The fishermen strongly believed that tides always give signals to the predicting weather conditions. In case of bad weather conditions, the fishermen keep their boats little away from the sea water to save their marine properties.

\section{CONCLUSION}

The fishing is one of the traditional occupation and fishing communities are solely depending on this activity for the livelihood. The distribution of nets, boats and carry these items for catching fish in appropriate season/months to make easy access of fishing expeditions. Based on the availability of fish varieties, fishermen carry these nets and perform their work activity with the help of fishermen knowledge. This knowledge further helps them to identify the fish locations and its availability at the different fishing grounds by employing variety of fish nets. And, the fishermen are expertised in identifying variety of fishes at the different locations to trace the fish behavior with the help of indigenous knowledge. However, the fishermen observed that the recent changes in the fishing activities over the period after introduction of modern methods in fishing. In addition, the climatic variations are also observed by the fishermen to impact on their knowledge practices.

\section{RECOMMENDATIONS}

The study is based on the ethnographic fieldwork which has been conducted among the fishing communities of north coastal Andhra Pradesh. The study shows that the fishermen communities

Anthropologist, 44(1-3): 1-9 (2021) 
are having lower level literacy rate and variety of occupational risks among them. In fact, it is needed to understand how fishing communities are coping with occupational difficulties by employing their indigenous knowledge practices while fishing engagement. Further, the local government should promote community developmental programmes for their welfare. And, it is also required to document the indigenous knowledge of the fishermen communities to protect their heritage and culture by promoting welfare and wellbeing.

\section{ACKNOWLEDGMENT}

I thank Prof. Vijaya Prakash and Prof. Jai Kishan for their support during the preparation of this work. I thank fishing community members who supported during the fieldwork in the studied areas. I also thank anonymous reviewers for their comments which helped to strengthen this paper.

\section{REFERENCES}

Acheson James M 1981. Anthropology of fishing. Journal of Annual Review of Anthropology, 10: 275-316.

Acheson James M 1988. The Lobster Gangs of Maine. Hanover and London: University Press of New England.

Acheson James M 2003. Capturing the Commons: Devising Institutions to Manage the Maine Lobster Industry. Hanover and London: University Press of New England.

Barth Fredrik 1966. Models of Social Organization. Royal Anthropological Institute, Occasional Papers 23.

Chandana Sarma 2018. Indigenous knowledge and fishing in Assam, Man in India, 98(2): 385-394.

Dalibandhu Pukkalla, Rama Mohan KR 2021. Local knowledge and marine livelihoods among the South Indian fishing community. Journal of Asian and African Studies, 56(3): 549-557.

Dalibandhu Pukkalla, Sharma BV 2020a. Divinatory functionaries and expansion of healing systems among the South Indian Fishing Community. Man In India, 100(1-2): 125-135.
Dalibandhu Pukkalla, Sharma BV 2020b. Transmission and erosion of local knowledge practices in a fishing village in South India. Journal of Ecological Anthropology, 22(1): 34-42.

Dalibandhu Pukkalla, Sharma BV 2018. Occupational health risks and etiologies among the Jalari community of Northern District of Andhra Pradesh, India. Journal of Human Ecology, 61(1-3): 64-71.

Firth Raymond 1946. Malay - Fishermen: Their Peasant Economy. London: Kegan, Paul, Trench Trubner \& Co., Ltd.

Hocart Arthur Maurice 1937. Fishing in Eddystone Island. The Journal of the Royal Anthropological Institute of Great Britain and Ireland, 67: 33-41.

Johannes Robert E 1978. Traditional marine conservation methods in ocean and their demise. Annual Review of Ecological Systems, 9: 349-364.

Kodanda Rao M 1975. Family and Kinship among Jalaris of Coastal Andhra. PhD Thesis, Unpublished. Visakhapatnam: Andhra University Library.

Madhanagopal D, Pattanaik S 2020. Exploring fishermen's local knowledge and perceptions in the face of climate change: The case of coastal Tamil Nadu, India. Environment, Development and Sustainability, 22: 3461-3489.

Malinowski Bronislaw 1931. Culture. In: Encyclopedia of the Social Sciences. Volume 4, pp.621-645. New York: Macmillan.

Mathur PRG 2008. Ecology, Technology and Economy: Continuity and Change among the Fisherfolk of Kerala. New Delhi: Rawat Publications.

Nuckolls William Charles 1996. The Cultural Dialectics of Knowledge and Desire. Wisconsin, USA: University of Wisconsin Press.

Prasada Rao DL, Yatiraj Kumar R 1984. Impact of mechanization on a traditional economy: A case study of fishermen in Visakhapatnam. Man Life, 10 (1 and 2): 31- 42.

Raychaudhuri B 1980. The Moon and Net: Study of a Transient Community of Fishermen at Jambudwip. Calcutta: Anthropological Survey of India.

Sridevi C 1989. The Fisherwoman Financier: A study of status-roles nexus in peasant community. Economic and Political Weekly, 29: 6-9.

Subba Rao Nune 1986. Economics of Fisheries: A Case Study of Andhra Pradesh. New Delhi: Daya Publishing House.

Suryanarayana M 1977. Marine Fisherfolk of North East Coastal Andhra Pradesh. Calcutta: Anthropological Survey of India.

Paper received for publication in June, 2021

Paper accepted for publication in August, 2021 